\title{
Psychiatric Crisis Intervention in Tunbridge Wells
}

ShIRley M. Smout, Consultant Psychiatrist, Hellingly Hospital, East Sussex; Mary ScotT, Nursing Officer, Community Psychiatric Nursing Service, Tunbridge Wells; Paul Fisher, Senior Social Worker, Tunbridge Wells Division of Social Services

The concept of crisis intervention and the various types of services available for dealing with crises affecting the individual or the family have been well set out in the publication from Napsbury Hospital. ${ }^{1}$ We would agree with Ratna's statement in that publication that crisis intervention is not only a system of delivering emergency psychiatric care, but also a set of techniques which utilizes a crisis to therapeutic advantage. It was with this in mind that a pilot study was launched in April 1979 for a crisis intervention service in Tunbridge Wells, Kent.

The catchment area has a population of 46,000 over the age of 16 years and is the remit of one consultant psychiatrist working 25 miles from the base hospital, which is in a separate health authority. Social work involvement was provided by experienced, authorized social workers from the local division. A psychiatric day treatment unit was already established at a general hospital in Tunbridge Wells and provided the base for the district community nursing service.

The objective was to make available, on request, a crisis team consisting of nurse, social worker and psychiatrist, who would visit the client at home within two hours of receiving the call. This service was to be an additional health-based service, available to GPs in the catchment area and to the police surgeon at Tunbridge Wells. Due to the limited number of personnel available, the service operated between 9 am and $5 \mathrm{pm}$, Monday to Friday. Requests for the crisis team were put through to the unit secretary, who had a rota of available personnel and contacted them using a radiotelephone service. The members met at an arranged time at the unit to screen available information prior to the visit. Requests for the service were not vetted in any way and al calls were accepted and responded to, unless there appeared to be overwhelming reasons for suggesting an alternative approach. No attempt was made to match team members to either each other or to the particular client; rather the aim was to build up a group of therapists capable of working together in various combinations.

From the outset it was normal practice to visit the client and available family members at home so that the presenting problem could be looked at in context. The aim was to assess the current problems, to identify precipitating factors, to obtain relevant past and present information and to focus on family interaction. The overall intent was a short-term intervention to alleviate the present crisis and to initiate further therapy in a separate setting, if appropriate. Where necessary the team arranged hospital admission. Towards the end of the session, team members would 'break' to make decisions and plan a therapeutic strategy which would then be put to the family.

At a later date the service was made available for patients in the general hospitals in the area.

An independent research worker made a study of the first six months of the new service, comparing it with the domiciliary visiting, community psychiatric nursing and outpatient services for the same psychiatric team. ${ }^{2}$ Results confirmed other studies 3,4 in showing an excess of female referrals to all services and extending over all diagnostic categories, except for addictions and marital problems. Of the total 359 referrals, 25 were to the crisis intervention service, an average of one per week. Seven out of every 10 referrals were to the traditional domiciliary and out-patient services, but nevertheless, the newer crisis intervention and community nursing services were handling three out of 10 referrals between them. Personality disorders and neuroses accounted for six out of every 10 referrals and crisis intervention, out-patient and community nursing services all seemed to be seeing the same range of problems. The domiciliary visiting service saw a higher proportion of psychoses and organic states than expected.

Although figures at this period were low, the referral levels of GPs were analysed. Average referrals to all services were 13.3 per practitioner. However, there were considerable differences between members of the same practice. Out of 27 doctors, 10 had not used crisis intervention by the end of the first six months. The pattern of referral seems to be an individual matter unrelated to actual incidence.

Forty per cent of cases seen at domiciliary visits were admitted to hospital, compared with 20 per cent of those seen by the crisis intervention service; the other two services had minimal admissions. This masked the real situation, in that a significant number of admissions were not included in the statistics for any of the four services examined. Out of 50 admissions, 20 were in fact planned admissions from various other sources.

A subjective assessment by the crisis intervention team found that the greatest benefit of setting up this service was the improvement in relationships between the various disciplines. Communication and a means of working towards a satisfactory conclusion in difficult cases had evolved and all workers felt more relaxed and able to interact and put their own point of view without feeling threatened. The team felt that it was much less stressful and more effective for members to respond to crises by means of such a coordinated service.

The service was assessed by the team after two years of operation and figures were compared with those for the domiciliary service. There were 46 referrals to crisis inter- 
vention in 1979/80 and 57 referrals in 1980/1, an average of about one per week. Referrals to the domiciliary service were about twice this number, with 95 during the twelve months of 1980 and 105 in the twelve months of 1981 . The consultant member started in post in January 1978, and the figures for the year prior to crisis intervention revealed that 125 domiciliary visits were done for a population comprising Tunbridge Wells and an additional 20,000 in East Sussex. The crisis intervention service has therefore not resulted in any significant reduction in requests for domiciliary visits; rather the community approach overall is leading to an increase in home assessments of all types, and there is a suggestion that referrals are still, if anything, increasing.

The admission rates from each service are interesting. Whilst the greatest number still comes via the domiciliary service, the percentage of crisis intervention clients admitted has been rising, with figures of 20 per cent in 1979, 22 per cent in 1980 and 35 per cent in 1981 . In contrast, the percentage admissions from the domiciliary service has been falling, with rates of 40 per cent in 1979,30 per cent in 1980 and 24 per cent in 1981. In other words, crisis intervention seems to be achieving what it set out to do, that is provide a service for urgent problems in the community and admission to hospital where appropriate.

The other figure of interest concerns the age range of referrals to each service. Eighty-eight per cent of referrals to crisis intervention were under 65 in 1979,87 per cent in 1980 and 77 per cent in 1981. Compared with these, 62 per cent of referrals for domiciliary visits were under 65 in 1979, 45 per cent in 1980 and 38 per cent in 1981. Thus crisis intervention does not seem to be seen as the appropriate referral service for the majority of the elderly by GPs, who use the domiciliary service instead.

The hospital admission figures for the Tunbridge Wells area show 146 admissions for $1977 / 8$ (that is the year prior to the consultant taking up post), 120 admissions for $1978 / 9$ and 100 admissions for $1980 / 1$. It is impossible to say whether this is significant in relation to the crisis intervention service. Looking at admissions for $1980 / 1,45$ patients came via the crisis intervention and domiciliary services, out of a total of 100 . This does suggest that crisis intervention, in the context of the present Tunbridge Wells service, has little potential to alter admission levels drastically. Almost all the remaining $\mathbf{5 5}$ admissions for the year were planned.

In September 1981 a grant was obtained to enable an independent research worker ${ }^{3}$ to interview the 30 GPs who had access to the crisis intervention service. All but three had been interested in the idea of a crisis service. These three were not against the service but were sceptical as to whether it would get off the ground. At interview, 25 were keen for the service to continue, two were indifferent and two were against continuation, wanting other facilities and feeling that it was wasted time.

Twenty-five had used the service at least once, the average was five calls per doctor. Four had not used the service because of lack of 'suitable clients' presenting in working hours. The service was seen as most relevant for potential admissions and urgent cases, about two-thirds of practitioners saying that they considered using the service in these situations. Avoiding admission to hospital, obtaining advice or an alternative opinion were, however, also considered important. The service was seen as relevant across a wide spectrum of social and psychiatric crises, including psychoses, personality difficulties, and social and family crises. Most GPs felt that the service could be useful in helping with problems and crises in the elderly, but 11 felt that the service was not suitable for this patient group.

Over half the GPs welcomed the team-based aspect of the service. A small number viewed the service primarily as a means of arranging an urgent visit from the psychiatrist. Just under half those who had used the service had found it very effective in dealing with crisis situations and about the same number had found it quite effective. However, only about half considered that the service was economic in terms of use of time and professional expertise.

The main advantages of the service over other psychiatric services were thought to be the team's speed of response to a crisis call and the provision of simuitaneous access to all services and disciplines. Other important aspects were that the team provided a sense that something was being done and an opportunity for the GP to share responsibility. Major disadvantages were that clients could feel overwhelmed by the number of people arriving and the fact that the service did not operate at night or over the weekend. In general, GPs were fairly confident that they had a clear idea of what was an appropriate referral to the team.

One patient had refused to be seen by the team and a third of the GPs had received adverse comments from the patient or family after crisis intervention visits; three patients had asked to be referred elsewhere. Adverse comments ranged from too personal questioning to feeling overwhelmed, lack of constructive help, and a relative not understanding what was happening.

\section{Some practical implications}

It is important that the team has adequate experience and knowledge to take decisions without immediate referral to seniors, otherwise decisions are delayed and become ineffective. The team should meet, visit and introduce itself as a team and give a clear explanation of its role and how it likes to work. At the same time, each member carries responsibility in his own area of expertise. Whilst team members will break and discuss the clinical situation and work towards a joint decision, occasionally, for example, a medical member may have to pinpoint for the team the necessity for more extensive medical assessment or may have to insist on individual time to assess suicide risk, etc. The same applies to other team members. Two of the most difficult cases were clients who eventually proved to have 
frontal lobe tumours. In retrospect, the team, with its social orientation, may have made it more difficult to focus on, and exclude, possible organic pathology at an early stage.

\section{REFERENCES}

'RATNA, L. (1976) The practice of psychiatric crisis intervention. League of Friends, Napsbury Hospital, 1.

${ }^{2}$ Cooper, J. (1979) Crisis Intervention in Tunbridge Wells and
Southborough: A study of the first six months of operation. Unpublished paper.

${ }^{3}$ Shepherd, M., CoOper, B., Brown, A. \& Kalton, C. (1966) Psychiatric Illnesses in General Practice. London: Oxford University Press.

4 GROVE, W. \& TUDOR, J. (1973) Adult sex roles and mental illness. American Journal of Sociology, 78, 812-35.

${ }^{5}$ HANNAL, P. (1981) Crisis intervention in Tunbridge Wells and Southborough: Views of general practitioners in the area. Unpublished paper.

\section{Formulating a Psychiatric Case}

F. T. VArghese, Consultant Psychiatrist, Royal Park Hospital, Melbourne, Australia, and G. W. MzLlsop, Professor of Psychological Medicine, Wellington Hospital, Wellington, New Zealand

Interest in the process of formulating a psychiatric case continues unabated (Greenberg et al, 1982). We report the findings of a brief survey conducted in an attempt to begin publicizing examiners' expectations in this contentious area.

In Australia and New Zealand formulations are regarded as an important part of case writing for the Membership examinations (RANZCP, 1980). Neither the English viewpoint eschewed in Scribe's Column (Royal College of Psychiatrists, 1979) nor the Canadian report of Ben-Aron and McCormick (1980) appear sufficiently detailed to guide teachers and psychiatric trainees.

To provide background information which would assist clinicians, supervisors and trainees to develop their views and to stimulate further debate, we set out to collect the views of psychiatric postgraduate examiners on the optimum format and content of a formulation, and to establish the consistency of their views.

\section{Method}

Twelve 'statements' about patients were collected. Six of these were written for the purpose by psychiatric registrars. The material written under the heading 'Formulation' in completed postgraduate case histories comprised the remainder. All twelve were rigorously rendered nonidentifiable.

These twelve formulations (A to $\mathrm{L}$ ) were sent to $\mathbf{4 0}$ senior Australian and New Zealand psychiatrists who have examined postgraduates. Twenty of these were frequent examiners (over at least three years) and 20 were occasional examiners. All were asked to: (a) give each formulation a mark out of ten; (b) complete a structured questionnaire for each formulation which they had marked as better than 6 out of 10 , or worse than 4 out of 10; (c) make any comments they wished about the concept or format of formulating.

The questionnaire allowed the examiners to record as 'good', 'unremarkable', or 'a problem area' aspects such as content (including history, premorbid personality, mental state and physical examination), actiological understanding, diagnosis, management and prognosis. They were also invited to comment on the style, use of English and clinical logic of the formulations.

For each examiner the formulations were ranked from 1 to 12 according to the mark given. The mean rankings were determined and the Kendall Coefficient of Concordance (W) calculated (Siegel, 1956). An attempt was made to summarize the viewpoints conveyed.

\section{Results}

Twenty-four (60 per cent) examiners replied to our request, of these 12 were frequent and 12 were occasional examiners. Overall, the ratings of the examiners are signifcantly correlated $(P<0.001)$. The strength of this is greater for the frequent than for the occasional examiners (although both at the 0.001 level).

The formulation (Appendix) which averaged the highest ranking was rated highly in most areas except 'dynamic understanding', with 41 per cent of examiners commenting adversely on this aspect. The presentation of data and the proposed management were highly rated, but assessors were evenly divided on prognosis, use of English, style and clinical logic. Typical favourable comments centred around the formulation being concise, comprehensive and well set out, without 'jargon'. Unfavourable comments related to insufficient attention to dynamic and social factors and discussion of differential diagnoses.

When the three lowest-ranked formulations were grouped together, it was clear that almost all aspects were rated poorly by between 50 per cent and 75 per cent of the examiners. Inadequate data, unjustified diagnoses and differential diagnoses and management stood out as particular problem areas. On the other hand, the use of English, style and clinical bgic (or lack of it) were not notably criticized.

Typical unfavourable comments in one of these formulations were as follows: 'so condensed as to be incomprehensible'; 'no understanding of multiple factors to explain the 